\title{
Innate, adaptive, and cell-autonomous immunity against Toxoplasma gondii infection
}

Miwa Sasai $^{1,2}$ and Masahiro Yamamoto ${ }^{1,2}$

\begin{abstract}
Hosts have been fighting pathogens throughout the evolution of all infectious diseases. Toxoplasma gondii is one of the most common infectious agents in humans but causes only opportunistic infection in healthy individuals. Similar to antimicrobial immunity against other organisms, the immune response against $T$. gondii activates innate immunity and in turn induces acquired immune responses. After activation of acquired immunity, host immune cells robustly produce the proinflammatory cytokine interferon- $\gamma$ (IFN- $\gamma$ ), which activates a set of IFN- $\gamma$-inducible proteins, including GTPases. IFN-inducible GTPases are essential for cell-autonomous immunity and are specialized for effective clearance and growth inhibition of $T$. gondii by accumulating in parasitophorous vacuole membranes. Recent studies suggest that the cell-autonomous immune response plays a protective role in host defense against not only $T$. gondii but also various intracellular bacteria. Moreover, the negative regulatory mechanisms of such strong immune responses are also important for host survival after infection. In this review, we will discuss in detail recent advances in the understanding of host defenses against $T$. gondii and the roles played by cell-autonomous immune responses.
\end{abstract}

\section{Introduction}

Toxoplasma gondii is a protozoan parasite belonging to the phylum Apicomplexa and can infect almost all warmblooded animals. One-third of the human population is reported to be infected with $T$. gondii, rendering it classifiable as a common pathogen. Despite the high infection rate in humans, $T$. gondii infection is opportunistic in individuals with normal immunity. Conversely, in immunocompromised individuals, such as AIDS patients, those undergoing chemotherapy and immunosuppressed tissue transplant patients, infection and reactivation can lead to chronic infection with $T$. gondii, potentially resulting in lethal encephalitis (Fig. 1a) ${ }^{1}$. The life cycle of T. gondii is composed of two reproductive stages: sexual and asexual stages in definitive and intermediate hosts, respectively. T. gondii can establish sexual reproduction only in the epithelium of

\footnotetext{
Correspondence: Masahiro Yamamoto (myamamoto@biken.osaka-u.ac.jp) 'Department of Immunoparasitology, Research Institute for Microbial Diseases, Osaka University, Osaka, Japan

${ }^{2}$ Laboratory of Immunoparasitology, WPI Immunology Frontier Research Center, Osaka University, Osaka, Japan
}

the digestive tract in cat species. On the other hand, the parasite asexually proliferates in all warm-blooded animals, including humans and livestock animals. Infection of intermediate hosts is mediated through oral digestion of cysts that are formed in tissues of intermediate hosts or oocysts that are formed in cat species. Oocysts or bradyzoites contained in the ingested cysts invade the host intestinal epithelium via the digestive tract wall and transform into tachyzoites. Subsequently, tachyzoites asexually proliferate several times and expand the infection in vivo. After the host detects $T$. gondii infection and activates the immune system, $T$. gondii tachyzoites transform into bradyzoites and form cysts in the central nervous or muscle tissues. The tissue cyst is a membranous structure containing lectins, such as $\mathrm{N}$-acetylglucosamine and $\mathrm{N}$-acetylgalactosamine, and can survive without being attacked by the host immune system (Fig. 1b) ${ }^{2}$. T. gondii actively infects host cells and resides in special membranous structures called parasitophorous vacuoles (PVs), where T. gondii is able to proliferate. If the PV is disrupted, $T$. gondii cannot survive within host cells. Host immune cells not only 

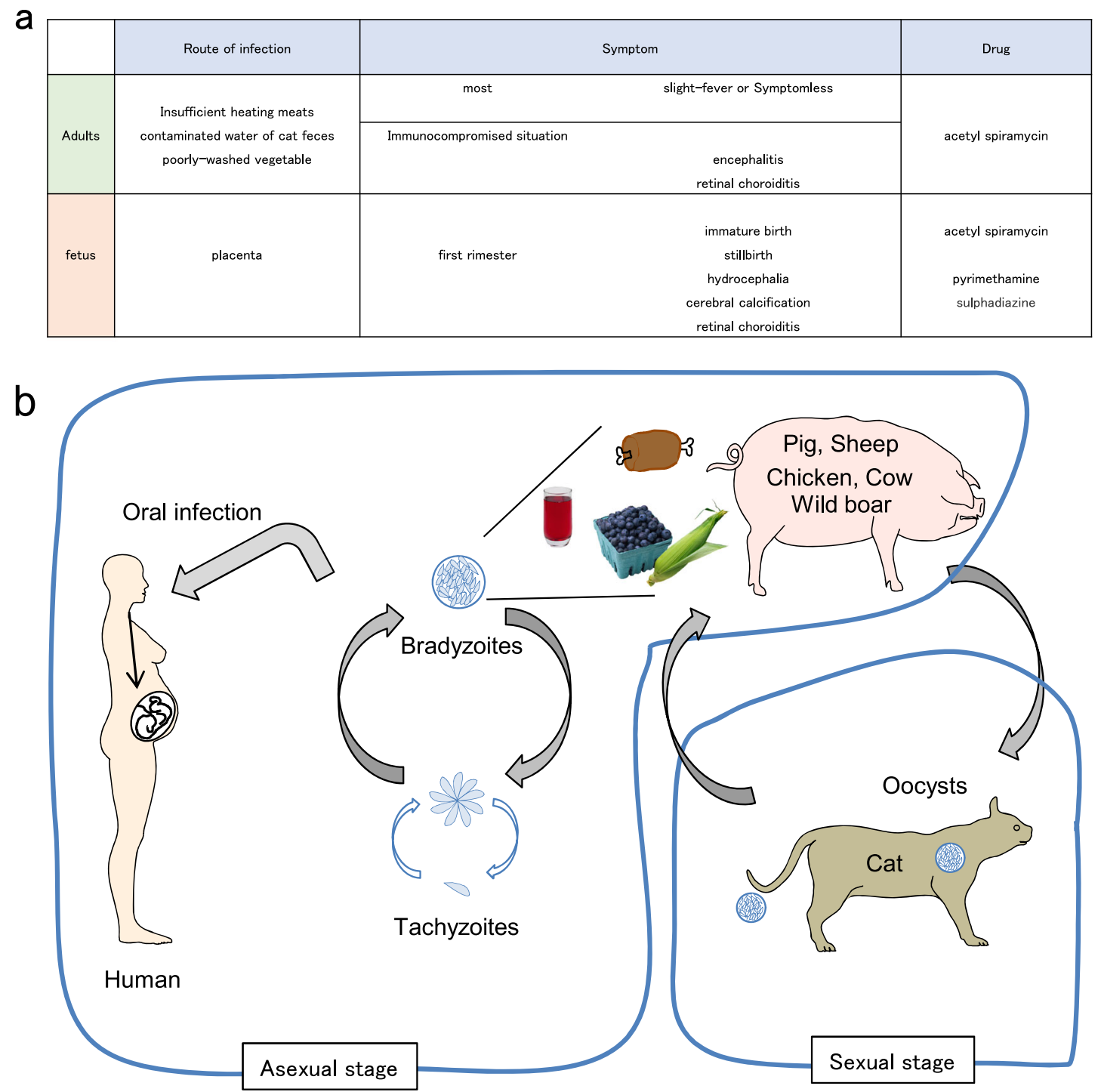

Fig. 1 Toxoplasmosis and the life cycle of $\boldsymbol{T}$. gondii. a Symptoms following T. gondii infection in humans. $\mathbf{b}$ Life cycle of T. gondii. When infected with $T$. gondii, felines shed oocysts in feces into the environment. The oocysts contaminate water, soil, fruits and vegetables from gardens and are easily taken in by humans and other intermediate hosts.

recognize T. gondii-derived ligands to activate acquired immunity but also destroy the PVs to remove proliferating T. gondii and ultimately block parasite expansion in vivo. However, T. gondii secretes various virulence factors into host cells to inhibit host acquired and innate immune responses. In this review, we will introduce recent findings regarding host defenses against $T$. gondii and the counterdefense mechanisms of the parasite.

\section{Innate and acquired immune responses against T. gondii infection \\ Recognition of $T$. gondii by innate sensors}

Innate immunity is the first line of host defense that responds immediately and detects pathogen infection via pattern recognition receptors (PRRs), such as Toll-like receptors (TLRs), Nod-like receptors, and C-type lectins. Ligand recognition by PRRs induces proinflammatory cytokines, including TNF- $\alpha$, interleukin- 6 (IL-6) and IL12 , and plays a role in the subsequent activation of immune responses ${ }^{3-5}$. The production of IL-12, which is important for the activation of acquired immunity in response to $T$. gondii infection, is severely impaired in MyD88-deficient mice ${ }^{6}$. MyD88 is an adaptor molecule that acts downstream of TLRs and the IL-1 receptor family members. TLRs are a group of 10 and 13 members in humans and mice, respectively, that differentially recognize pathogen-derived components and are involved in early pathogen detection. In mice, TLR11 has been 
shown to recognize $T$. gondii-derived profilin-like molecules, which are essential for parasite invasion of host cells. IL-12 is then induced in a MyD88-dependent manner ${ }^{7,8}$. Furthermore, TLR12 has been reported to detect profilin-like protein through its homodimer or as a heterodimer with TLR11, also resulting in IL-12 production $^{9}$. In addition, IL-12 production in response to $T$. gondii infection has been shown to be reduced in mice that are deficient in the chemokine receptor CCR5. $T$. gondii-derived cyclophilin-18 (TgCyp18) is detected by CCR5 on dendritic cells (DCs), thus stimulating IL-12 expression ${ }^{10}$. CCR5 induces IL-12 production through activation of the G protein $\alpha$ I family and MAPK, suggesting that this CCR5-dependent signaling pathway plays an important role in the immune response against $T$. gondii $^{11,12}$. Since CCR5 is mainly expressed on DCs, macrophages, $\mathrm{T}$ cells and microglia, CCR5-dependent production of inflammatory cytokines may be implicated in the induction of encephalitis. Moreover, given that CCR5-deficient mice show reduced embryonic lethality during pregnancy in comparison with that of wild-type mice, CCR5-dependent inflammation may be involved in fetal lethality and miscarriage when pregnant women undergo primary infection with $T$. gondii ${ }^{13}$. Both profilinlike protein and TgCyp18 stimulate IL-12 production by CD $8 \alpha^{+}$and CD $8 \alpha^{-}$DCs. Activation of TLR11 and TLR12 leads to IL-12 expression in plasmacytoid DCs. However, although $T$. gondii infection in mice causes IL-12 production in manners that are dependent on TLR11/TLR12 or CCR5, humans do not possess TLR11 and TLR12. How can humans produce IL-12 in response to $T$. gondii infection? Human monocytes produce IL-12 following $T$. gondii infection, suggesting that TLRs other than TLR11 and TLR12 may play a role in the detection of $T$. gondiiderived ligands in humans. TLR11 and TLR12 form heterodimers or homodimers that are expressed in endosomal compartments of the host cell cytosol. Since the ligand recognition mechanism of TLR11 and TLR12 in endosomes is similar to that of human TLR3, TLR7 and TLR9, which recognize nucleic acids, these TLRs, as well as TLR8, might be stimulated by $T$. gondii-derived nucleic acids in the endosome to induce IL-12 expression ${ }^{14}$. Although human CCR5 associates with TgCyp18, it is unclear whether CCR5 is critical for the production of IL12 following $T$. gondii infection in humans ${ }^{15}$. Further investigation to assess the function of CCR 5 on responses against $T$. gondii in humans will be required in the future. Furthermore, unidentified PRRs may be involved in the detection of $T$. gondii infection to produce IL-12 in a manner that is independent of MyD88 and CCR5. Future studies analyzing the machinery involved in $T$. gondii detection and IL-12 production in humans will be of interest. It has been very recently reported that human cells infected with $T$. gondii release S100A11 in a manner that is dependent on caspase-1, resulting in CCL2 production through receptor of AGE (RAGE) and monocyte chemotaxis to the infected site ${ }^{16}$. Thus, various factors released from infected cells strongly affect the subsequent activation of immune responses.

\section{Immune response at the infection site of $T$. gondii}

$T$. gondii usually infects humans and animals through oral infection. When $T$. gondii cysts and oocytes present in undercooked meats or on unwashed fresh vegetables are ingested, the intestinal mucosa is the first place in the host body that encounters the organism. Innate lymphoid cells (ILCs) are a recently identified lymphocyte population consisting of three groups based on their functional characteristics $^{17}$. Group 1 ILCs (ILC1s) consist of ILC1 and conventional natural killer (NK) cells. Activation of ILC1s leads to the production of Th1 cytokines, such as IFN- $\gamma$ and TNF- $\alpha{ }^{18}$. Group 2 ILCs (ILC2s) produce IL-4, IL-5, IL-9 and IL-13, which are Th2 cytokines. Group 3 ILCs (ILC3s) produce IL-17A and IL-22. When T. gondii infects orally, ILC1s produce IFN- $\gamma$ and TNF- $\alpha$ in response to $T$. gondii infection ${ }^{19,20}$. In addition, the number of ILC3s in the lamina propria of aryl hydrocarbon receptor (Ahr)-deficient mice is decreased, resulting in overactivation of CD4 $\mathrm{T}$ cells in response to T. gondii infection. Thus, ILC3s and ILC1s are indirectly involved in the host defense against $T$. gondii ${ }^{21}$. Notably, the mechanism by which $T$. gondii infection activates ILCs is completely unknown. Moreover, the molecular mechanism by which ILC activation leads to the subsequent induction of acquired immune responses remains enigmatic at present.

\section{Acquired immune responses following sensing of $T$. gondii}

The activation of DCs and macrophages in response to T. gondii infection results in the production of various cytokines, such as IL-1 $\beta$ and TNF- $\alpha^{22-24}$. Simultaneous administration of TNF- $\alpha$ and IL- $1 \beta$ or IL- $1 \alpha$ in mice is protective against $T$. gondii infection in a manner that is highly dependent on IL- $12^{25}$. IL-12 produced during innate immune responses in DCs, plasmacytoid DCs, and macrophages stimulates proliferation of NK cells, $\mathrm{CD}^{+}{ }^{+} \mathrm{T}$ cells, and cytotoxic $\mathrm{CD} 8^{+} \mathrm{T}$ cells, resulting in massive IFN- $\gamma$ production $^{26,27}$ (Table 1). In particular, $\mathrm{CD} 8 \alpha^{+}$DCs migrating into local sites of $T$. gondii invasion detect the infection and produce IL-12, which further stimulates NK cells to generate IFN- $\gamma$ in the bone marrow $^{28}$. When severe combined immunodeficient (SCID) mice, which lack B and T lymphocytes, are infected with $T$. gondii and are treated with IL-12, systemic IFN- $\gamma$ levels and eventual survival rates are increased, suggesting that IFN- $\gamma$ derived from NK cells plays an important role in the host defense against $T$. gondii during the acute phase of $T$. gondii infection 
Table 1 List of major cytokines that are produced by T. gondii infection and the effect of each cytokine.

\begin{tabular}{|c|c|c|c|}
\hline Cytokines & Producing cell & Effects & Refs. \\
\hline \multirow[t]{3}{*}{$\mathrm{IL}-12$} & $\mathrm{CD}_{8} \mathrm{a}^{+}$or $\mathrm{CD} 8 \mathrm{ar} \mathrm{CDCS}$ & Activate and stimulate proliferation of NK cells, CD4 T cells and CD8 T cells & $6-8$ \\
\hline & Macrophages & & \\
\hline & $\mathrm{pDCs}$ & & $9,10,25$ \\
\hline \multirow[t]{5}{*}{$\mathrm{IFN}-\gamma$} & $\mathrm{ILC} 1 \mathrm{~s}$ & Induce cell-autonomous immune responses & $19,20,24$ \\
\hline & CD4 T cells & iNOS induction & $26-28$ \\
\hline & CD8 T cells & IDO production & 29,32 \\
\hline & NK cells & & \\
\hline & T-bet ${ }^{+}$Foxp3-Th1 cells & & \\
\hline TNF-a & Macrophages DCs & Induce other inflammatory proteins & $22-24$ \\
\hline $\mathbb{L L}-1 \beta$ & Macrophages & Induce other inflammatory proteins & $22-24$ \\
\hline \multirow[t]{3}{*}{$\mathrm{IL}-10$} & Ly6ChighCCR2 + monocytes & Suppress inflammation to prevent toxoplasma encephalitis & $43,47,48$ \\
\hline & B cells & Suppress hyper inflammation & $49-51$ \\
\hline & T-bet+Foxp3-Th1 cells & & $52-54$ \\
\hline \multirow[t]{3}{*}{ IL-27 } & Myeloid cells & Required for resistance to chronic toxoplasmic encephalitis & $45,46,55$ \\
\hline & & Induce CXCR3, T-bet, Blimp1 and IL-10 expression & \\
\hline & & Inhibit Th17 development & \\
\hline \multirow[t]{2}{*}{ IL-33 } & Damaged cells & Induce CCL2 expression (proinflammatory) & 56 \\
\hline & Tissue injury & Induce IL-10 production by M2 macrophages (anti-inflammatory) & \\
\hline
\end{tabular}

in vivo ${ }^{29}$. Even when NK cells or $\mathrm{CD}^{+} \mathrm{T}$ cells are depleted by antibodies in wild-type mice, acute resistance to $T$. gondii infection is unaffected ${ }^{29}$. In contrast, mice lacking the common $\gamma$-chain and are thus devoid of NK cells and $\mathrm{CD}^{+} \mathrm{T}$ cells, lose acute resistance to $T$. gondii in conjunction with further $\mathrm{T}$ cell depletion by anti-CD4 antibody, resulting in high susceptibility and indicating that IFN- $\gamma$ produced by NK cells and $C D 4^{+} \mathrm{T}$ cells contributes to the host defense against $T$. gondii host ${ }^{29}$. In addition, a recent study demonstrated that neutrophils can produce IFN- $\gamma$ in TLR11-deficient mice that are systemically infected with $T$. gondii ${ }^{30}$. It is still unclear whether neutrophils produce IFN- $\gamma$ in wild-type mice. Moreover, it has been very recently reported that a new cell population called memory-phenotype cells (MP cells), which are pathogen-independent memory-phenotype CD4 T cells, produce IFN- $\gamma$ in response to IL-12 in $T$. gondii infection ${ }^{31}$. Thus, in addition to classical IFN- $\gamma$-producing cells, such as NK cells and T cells, new populations including ILC1s, neutrophils, and MP cells have been identified. However, the mechanism by which these classical and new IFN- $\gamma$ producing cells sequentially and mutually lead to IFN- $\gamma$ production remains to be elucidated.

Inducible nitric oxide synthase (iNOS) is an IFNinducible protein that is also upregulated by $T$. gondii infection. Although lack of iNOS in mice does not affect acute control of $T$. gondii infection, iNOS-deficient mice are highly susceptible during the late phase of infection, resulting in lethality due to increased parasite burden in the brain ${ }^{32,33}$. Nitric oxide generated by iNOS activation plays a role in the suppression of cerebral toxoplasmosis in the fetus. Notably, the effect of iNOS can be detected in C57BL/ 6 but not in BALB/C mice, since T. gondii encephalitis does not usually occur in BALB/c mice ${ }^{34}$. In addition, IFN- $\gamma$ also stimulates robust production of indoleamine 2,3-dioxygenase (IDO), which suppresses $T$. gondii growth in human fibroblasts, glioma cells, retinoblastoma cells, and macrophages ${ }^{24,35-38}$.

In addition to IL-12 production derived from DCs and macrophages, various types of host cells are involved in immune responses against $T$. gondii. When $T$. gondii infects a host, intestinal epithelial cells and peritoneal cells are primarily infected under physiological conditions. Antigen-presenting cells such as DCs and macrophages recognize $T$. gondii-derived factors, including virulence effectors, to produce chemokines such as CCL2 and CXCL2 $2^{39,40}$. Such chemokines induce migration of $\mathrm{Ly}_{6 \mathrm{C}}{ }^{\text {high }} \mathrm{CCR} 2^{+}$monocytes and neutrophils to the sites of infection ${ }^{41}$. These monocytes and neutrophils that are infected with $T$. gondii spread systemically, while expression of IFN- $\gamma$ and IFN-inducible proteins control the systemic infection ${ }^{42}$. However, 
Ly6C ${ }^{\text {high }} \mathrm{CCR} 2^{+}$monocytes also lead to the production of the anti-inflammatory cytokine IL-10, which is important for the suppression of Toxoplasma encephalitis, indicating that $\mathrm{Ly}_{6 \mathrm{C}}{ }^{\text {high }} \mathrm{CCR} 2^{+}$monocytes play both positive and negative roles in immune responses against $T$. gondii ${ }^{43}$. IL-10 produced by Foxp ${ }^{+} \mathrm{CD} 4^{+}$ $\mathrm{T}$ cells (Tregs) is important for the maintenance of immune homeostasis in uninfected hosts. In contrast, the suppressive mechanism mediated by Tregs is not required during infection. Thus, the number of Tregs is tightly controlled. When $T$. gondii infects a host, local concentrations of IL-2 are decreased, resulting in the inhibition of Tregs and activation of the Th1 immune response ${ }^{44}$. Since Th1 cells are more highly responsive to IL-2 than Tregs, a local reduction in IL-2 is thought to selectively decrease the Treg population ${ }^{44}$ (Table 1). IL27 and IL-12 synergize to limit IL-2 production during T. gondii infection ${ }^{45,46}$. However, immunosuppression after activation of Th1 cells is important for protection against excessive immunopathology in response to $T$. gondii infection. IL-10 and IL-22 belong to the IL-10 family of anti-inflammatory cytokines ${ }^{47}$. IL-10 is produced during not only the early phase post infection but also the chronic phase, especially in the brain ${ }^{48,49}$. IL-12 production is suppressed by Th2 cytokines, such as IL-4 and IL-10. Experiments using IL-10-deficient mice or wild-type mice treated with anti-IL-10 antibody demonstrate that IL-10 is produced by B cells and T-bet ${ }^{+}$Foxp $3^{-}$ Th1 cells to suppress hyper inflammation ${ }^{50-54}$. Tbet ${ }^{+}$Foxp $3^{-}$Th1 cells produce IL-10 as well as IFN- $\gamma$, in a 'self-regulation' mechanism ${ }^{54}$. Moreover, IFN- $\gamma$ stimulates T-bet expression in Tregs, subsequently leading to CXCR3 expression ${ }^{55}$. IL-27 stimulates not only T-bet and CXCR3 induction in Tregs but also IL-10 production. Blimp1 induces IL-10 to suppress inflammation and is also induced by $\mathrm{IL}-27^{56}$ (Table 1). IL-33 is also a proinflammatory cytokine that is induced by $T$. gondii infection and induces CCL2 expression in the brains of infected mice, leading to migration of $\mathrm{CCR}^{+}$monocytes and generation of the immune response against $T$. gondii. Conversely, IL-33 has been shown to amplify the Th2 response to suppress Th1 immunity and to stimulate IL-10 production by M 2 macrophages to activate Tregs ${ }^{57}$. Thus, the role of IL-33 in the immune response against $T$. gondii has two opposing aspects and hence requires further investigation to fully elucidate the molecular mechanisms involved (Table 1).

\section{Cell-autonomous immune responses against $T$. gondii infection}

IRG-mediated cell-autonomous immune responses

IFN- $\gamma$ produced by activated immune cells such as NK cells, ILC1 and T cells has been shown to play a pivotal role in anti-T. gondii immunity ${ }^{58}$. IFN- $\gamma$-stimulated cells express hundreds of IFN-stimulated genes, including GTPase family members. Among them, a recent extensive analysis of p47 and p65 GTPases revealed that these GTPases are strongly involved in the immune response against $T$. gondii ${ }^{59}$. Immunity-related GTPases (IRGs) (also called p47 GTPase) are family members whose molecular weights are $\sim 47-48 \mathrm{kDa}$. IRGs consist of IRGM, IRGA and IRGB family members, all of which possess GTP-binding domains, whose amino acid sequences can be classified into two major groups, GMSIRGs and GKS-IRGs ${ }^{60,61}$. GMS-IRGs consist of IRGM1 ${ }^{62}$, IRGM $2^{60}$ and IRGM $3^{63}$, and are mainly localized in the Golgi and $\mathrm{ER}^{64-66}$. The remaining IRGs, excluding the GMS-IRGs, are classified as GSK-IRGs, among which IRGA $6^{60}$ and IRGB6 ${ }^{67,68}$ are strongly involved in the immune response against $T$. gondii ${ }^{61}$. The mechanism by which GMS-IRGs regulate GKS-IRGs remains unclear. GMS-IRGs form heterodimers with GKS-IRGs to negatively regulate activation ${ }^{69}$. Regarding GKS-IRGs, it has been reported that IRGB10 and IRGD are recruited to the PVM following T. gondii infection, but it is not clear if they are critical in preventing T. gondii infection in vivo ${ }^{70}$. Moreover, it remains unclear whether GTPases are involved in responses against $T$. gondii and which are not involved because many GTPases are induced. Cells from mice lacking IRGM1 or/and IRGM3 showed decreased IRGA6 and IRGB6 protein expression levels and abnormal localization, resulting in impaired immune response against $T$. gondii ${ }^{71,72}$. PVs are specialized compartments generated by $T$. gondii in infected host cells. T. gondii resides within the PV and proliferates until transmission to the next host cells ${ }^{59,73}$. Among the IRGs, IRGA6, and IRGB6 in particular accumulate on the PV membrane (PVM) and destroy this structure ${ }^{69,74}$ (Fig. 2).

P65 GTPases are called guanylate-binding proteins (GBPs) and also play important roles in the host defense against T. gondii. Humans and mice possess 6 and 11 GBPs, respectively. In mice, GBPs form two gene clusters located on chromosomes 3 and 5 encoding $\mathrm{GBP}^{\text {chr } 3}$ and $\mathrm{GBP}^{\text {chr } 5}$, respectively ${ }^{75}$. GBP ${ }^{\text {chr } 3}$ consists of GBP1, GBP2, GBP3, GBP5 and GBP7. GBP1 and GBP7 have been shown to be involved in host defense against not only T. gondii but also Listeria monocytogenes and Mycobacterium bovis $\mathrm{BCG}^{76,77}$. Mice lacking $\mathrm{GBP}^{\text {chr3 }}$ are severely defective in the destruction of the T. gondii PVM in macrophages and exhibit dramatically high susceptibility to the pathogen ${ }^{78}$. In contrast, mice singly lacking GBP1 or GBP2 demonstrate a milder phenotype than that of $\mathrm{GBP}^{\text {chr3 }}$-deficient mice, indicating that $\mathrm{GBP}^{c h r 3}$ members other than GBP1 and GBP2 play a role in immune responses against $T$. gondii by compensating for the loss of GBP1 and GBP2 $2^{77,79}$ (Fig. 2). The precise functions of other GBPs such as GBP3 and GBP5 that are recruited to the PVM need to be clarified in the future. 


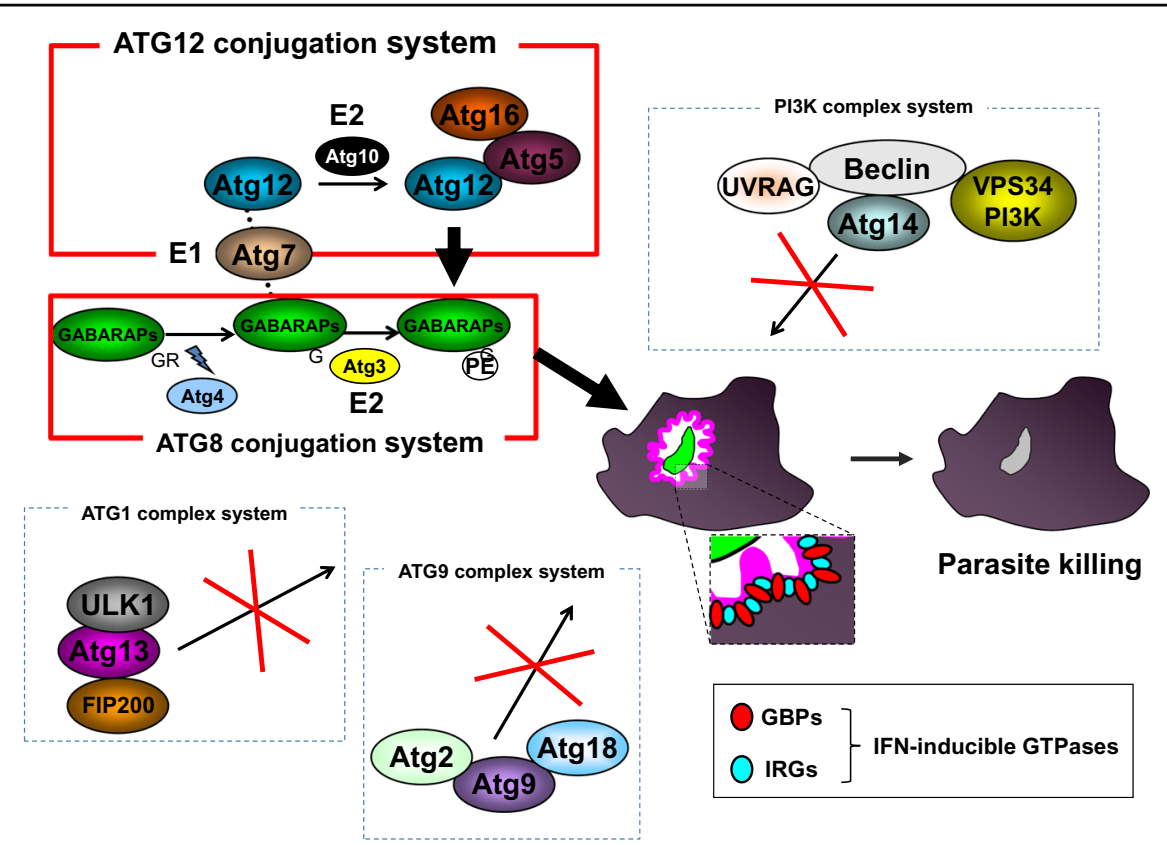

Fig. 2 Diagrams of cell-autonomous immune responses to $T$. gondii infection. IFN-inducible GTPases, such as GBPs and IRGs, accumulate on the T. gondii PVM and are important for its disruption. ATG family proteins are functionally divided into five systems, Atg12 conjugation, Atg8 modification, Atg1 complex, PI3K complex and Atg9 complex, which all play differentially critical roles in autophagy. Among them, IFN-inducible GTPase-dependent immunity requires Atg12 conjugation and Atg8 modification systems (marked red). Atg family members (marked red) regulate the localization of the GTPases that are involved in the destruction of PVMs. GTPases that are reportedly recruited to PVM are IRGs (Irga6, Irgb6, Irgb10 and $\operatorname{lrgD}$ ) and GBPs (GBP1, GBP2, GBP3, GBP5, GBP6, GBP7 and GBP9). Some GTPases on the PVM form pores on the PVM, resulting in destroying the replication location of $T$. gondii and preventing the expansion of infection.

\section{Ubiquitination of PVMs by IFN stimulation}

Although it is well documented that IFN-inducible GTPases play an important role in the immune response against $T$. gondii, the mechanism by which these GTPases are recruited to the PVM remains unclear. Analysis using a chimeric protein of GBP2 and GBP5 revealed that isoprenylation of GBP2 at the C-terminus is essential for GBP2 recruitment to the PVM. However, C-terminal isoprenylation of GBP2 is not sufficient to specifically recognize the $\mathrm{PVM}^{80}$. Given that host recognition of cytosolic bacteria involves ubiquitination of bacteria or bacteria-containing vacuoles, ubiquitination might be one way to distinguish host and T. gondii PVMs ${ }^{81,82}$. To date, modes of ubiquitination of $T$. gondii PVMs have been shown to be K48-linked, K63-linked and M1 linear types ${ }^{83,84}$. Analysis using cells lacking E3 ligases such as TRAF6 or TRIM21 demonstrated that these E3 ligases are involved in IFN- $\gamma$-induced ubiquitination of $T$. gondii $\mathrm{PVMs}^{85}$. However, given that the degree of ubiquitination and PV destruction in TRIM21-deficient cells is minimal, other E3 ligases, including TRAF6, may compensate for the loss of TRIM21. Analysis using cells with compound mutations in TRIM21 and TRAF6 will clarify whether these proteins compensate for each other or if other E3 ligases are involved. Recently, it has been reported that murine GBP1 and GBP2, which are members of the p65
GTPases, are ubiquitinated by TRIM $21^{86}$. However, the biological effects of ubiquitination of GBPs are completely unknown. Further research will clarify the function of ubiquitination following $T$. gondii infection.

In addition to the molecular mechanism by which IFNinducible GTPases are recruited to the T. gondii PVM, the biological significance of the recruitment of the ubiquitinbinding protein p62 to the PVM remains controversial. Similar to IFN-inducible GTPases, p62 plays an important role in $T$. gondii clearance ${ }^{87}$. Conversely, another report demonstrated that p62 is not required for IFN- $\gamma$-induced T. gondii clearance but is involved in the presentation of antigens located in the PV to activate $\mathrm{CD}^{+} \mathrm{T}^{+}$cells ${ }^{83}$.

\section{Regulation of cell-autonomous immune responses by autophagy-related genes}

Autophagy is a highly conserved biological response and is required for the maintenance of homeostasis by selfdigestion during periods of stress, such as nutrient starvation or embryonic development ${ }^{88}$. Autophagy is strictly regulated by autophagy genes and their products ${ }^{88}$. The mammalian homolog of ATG8 consists of the LC3 and GABARAP subfamilies. The LC3 subfamily comprises LC3A, LC3B and LC3C. In addition, the GABARAP subfamily consists of GABARAP, GABARAPL1 and GABARAPL2 (also known as Gate-16) ${ }^{89}$. LC3B is a 
homolog of yeast ATG8 and accumulates on the autophagosome to remove cytosolic pathogens. Subsequently, LC3B-coated autophagosomes fuse with the lysosome to digest the pathogens ${ }^{90}$. This particular process of autophagy is called xenophagy ${ }^{91}$. When cells are activated by CD40, LC3B also accumulates on T. gondii PVMs. Stimulation with CD40 induces physical interaction of the PVM and the lysosome, possibly leading to activation of PI3K3C, Rab7, and vATPase ${ }^{92}$. However, the recruitment of LC3B to the T. gondii PVM is controversial ${ }^{93}$. LC3B might be involved in the functionality of the IFN- $\gamma$ inducible anti- $T$. gondii response.

During autophagy, ATG5 and ATG12 form a complex to mediate the modification of phosphatidylethanolamine on the C-terminus of ATG8 ${ }^{94}$. Cells lacking ATG5, an essential autophagy protein, are also severely defective in IFN- $\gamma$-induced PVM destruction, which is essential for the inhibition of T. gondii proliferation ${ }^{95}$. In general, although the autophagosome is a doublemembraned structure, the T. gondii PV does not possess a double membrane, suggesting that the function of ATG5 in the immune response against $T$. gondii is independent of autophagy ${ }^{96}$. Subsequently, the GABARAP subfamily but not the LC3 subfamily plays an important role in the uniform distribution of GBPs in host cells to efficiently mediate host defense against T. gondii. GBPs are located on small vesicle-like structures $(\mathrm{VLSs})^{77}$. Among GABARAP subfamily members, Gate-16 in particular is involved in the uniform localization of GBP2-containing VLSs. GBP2containing VLSs aggregate in the cytosol and are dysfunctional in Gate-16-deficient cells. Mice lacking Gate16 specifically in the myeloid cell lineage are defective in GBP-dependent host defense against $T$. gondii in vivo ${ }^{42}$ (Fig. 2). Even Gate-16-deficient or Atg5-deficient cells showed dramatically increased $T$. gondii numbers, and parasite numbers in autophagy-deficient cells, such as cells lacking FIP200 and Atg9, were intact following IFN- $\gamma$-stimulation. The effects of xenophagy on immune responses against $T$. gondii are not extensive.

\section{Conclusion}

Innate and adaptive immune responses to $T$. gondii are being extensively studied using gene knockout mice. Among these analyses, cell-autonomous immunity involving IFN- $\gamma$-inducible GTPases, such as GBP and IRG provides us with a new concept that is distinct from previously established immunological ideas. Recently, cell-autonomous immunity has been shown to have a defensive function against pathogens other than $T$. gondii (Fig. 3). GBP1 is reported to be involved in the suppression of L. monocytogenes or M. bovis BCG infection ${ }^{76}$. GBP2 and IRGB10, another IRG member, accumulate on chlamydia-containing vacuoles in a manner that is

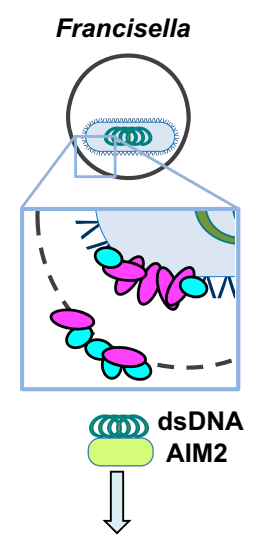

IL-1 / IL-18

Canonical Inflammasome
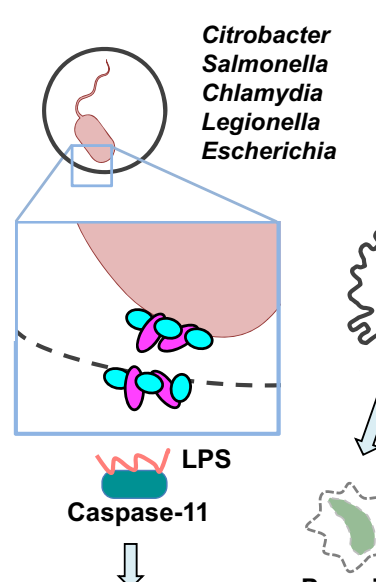

Parasite clearance

IL-1 $\beta$ / IL-1 $\alpha$ / IL-18

Non-canonical

Inflammasome

Toxoplasma gondii
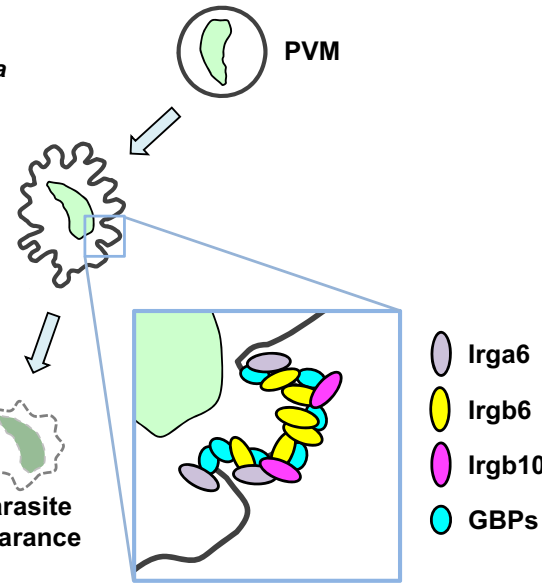

Fig. 3 IFN-inducible GTPase-dependent cell-autonomous immunity to intracellular pathogens. Francisella is Gram-negative bacterium that resides within a phagosome called the Francisella-containing phagosome (FCP), which is an initial vacuolar compartment. DNA from Francisella is somehow released into the cytosol and is recognized by AIM2, which is a cytosolic sensor that recognizes DNA, following the activation of the canonical inflammasome. Some bacteria, such as Salmonella and Citrobacter, are also Gram-negative bacteria and remain in the vacuoles in the host cytosol. LPS from these bacteria somehow binds to caspase-11 and then activates noncanonical inflammasomes. IRGs and GBPs accumulate on PVs of T. gondii, bacteria-containing vacuoles or bacterial cell membranes of various intracellular bacteria to eliminate pathogens and subsequently induce activation of the inflammasome. The function of each IRG and GBP on vacuoles is not fully understood. 
dependent on Atg3 and Atg5 ${ }^{97}$. In addition, GBP $P^{\text {chr3 }}$ is involved in LPS-induced caspase-11 activation that is induced by infection with Legionella, Salmonella or E. coli to inhibit bacterial growth by a mechanism of cell death known as pyroptosis ${ }^{98}$. Moreover, Chlamydia infection causes activation of the canonical inflammasome involving caspase-1 as well as the caspase-11dependent noncanonical inflammasome in a GBPdependent manner ${ }^{99}$. Furthermore, GBPs induced by IRF1 are important for AIM2-dependent detection of double-stranded DNA resulting from cytosolic Francisella proliferation ${ }^{100,101}$ (Fig. 3). IRGB10 is also involved in $\mathrm{GBP}^{\mathrm{chr}}$-dependent AIM2-induced activation of the inflammasome ${ }^{102}$. GBPs are required for noncanonical inflammasome activation mediated by outer membrane vesicles, which are released from Gram-negative bacteria and contain LPS ${ }^{103}$ (Fig. 3). Thus, given that GBPs and IRGs play various roles in regulating host defense and pathogen infection, it is reasonable to assume that pathogens establish counter-defense mechanisms. For instance, Shigella possesses an effector molecule called IpaH9.8 to inhibit GBP2-dependent immune responses against Shigella ${ }^{104,105}$. As such, the pathogen-mediated anti-"anti-pathogen immune response" should be further analyzed. In particular, the exact molecular mechanisms by which GBPs and IRGs mediate cell-autonomous immunity against $T$. gondii and other pathogens, including bacteria and viruses, will be of interest in the future.

\section{Acknowledgements \\ We thank M. Enomoto (Osaka University) for secretarial and technical assistance. This work is supported by the Research Program on Emerging and Re-emerging Infectious Diseases (JP19fk0108047) and the Japanese Initiative for Progress of Research on Infectious Diseases for Global Epidemic (JP19fm0208018) from the Agency for Medical Research and Development (AMED), the Cooperative Research Grant of the Institute for Enzyme Research, the Joint Usage/Research Center, Tokushima University, the Takeda Science Foundation, the Ohyama Health Foundation, the Naito Foundation, the Cell Science Research Foundation, the Mochida Memorial Foundation on Medical and Pharmaceutical Research, the Uehara Memorial Foundation, and the Research Foundation for Microbial Diseases of Osaka University.}

\section{Conflict of interest}

The authors declare that they have no conflict of interest.

\section{Publisher's note}

Springer Nature remains neutral with regard to jurisdictional claims in published maps and institutional affiliations.

Received: 28 March 2019 Revised: 29 October 2019 Accepted: 30 October 2019.

Published online: 11 December 2019

\section{References}

1. Howard, J. C., Hunn, J. P. \& Steinfeldt, T. The IRG protein-based resistance mechanism in mice and its relation to virulence in Toxoplasma gondii. Curr. Opin. Microbiol. 14, 414-421 (2011).
2. Bohne, W., Holpert, M. \& Gross, U. Stage differentiation of the protozoan parasite Toxoplasma gondii. Immunobiology 201, 248-254 (1999).

3. Sasai, M. \& Yamamoto, M. Pathogen recognition receptors: ligands and signaling pathways by Toll-like receptors. Int. Rev. Immunol. 32, 116-133 (2013).

4. Quinn, S. R. \& O'Neill, L. A. A trio of microRNAs that control Toll-like receptor signalling. Int. Immunol. 23, 421-425 (2011).

5. Beutler, B. et al. Genetic analysis of host resistance: Toll-like receptor signaling and immunity at large. Annu. Rev. Immunol. 24, 353-389 (2006).

6. Scanga, C. A. et al. Cutting edge: MyD88 is required for resistance to Toxoplasma gondii infection and regulates parasite-induced IL-12 production by dendritic cells. J. Immunol. (Baltimore, MD: 1950) 168, 5997-6001 (2002).

7. Plattner, F. et al. Toxoplasma profilin is essential for host cell invasion and TLR11-dependent induction of an interleukin-12 response. Cell Host Microbe. 3, 77-87 (2008).

8. Yarovinsky, F. et al. TLR11 activation of dendritic cells by a protozoan profilinlike protein. Science (New York, NY) 308, 1626-1629 (2005).

9. Koblansky, A. A. et al. Recognition of profilin by Toll-like receptor 12 is critical for host resistance to Toxoplasma gondii. Immunity 38, 119-130 (2013).

10. Aliberti, J. et al. CCR5 provides a signal for microbial induced production of IL-12 by CD8 alpha+ dendritic cells. Nat. Immunol. 1, 83-87 (2000).

11. Sher, A. et al. Induction and regulation of IL-12-dependent host resistance to Toxoplasma gondii. Immunol. Res. 27, 521-528 (2003).

12. Denkers, E. Y., Butcher, B. A., Del Rio, L. \& Kim, L. Manipulation of mitogenactivated protein kinase/nuclear factor-kappaB-signaling cascades during intracellular Toxoplasma gondii infection. Immunol. Rev. 201, 191-205 (2004).

13. Nishimura, M., Umeda, K., Suwa, M., Furuoka, H. \& Nishikawa, Y. CCR5 is involved in interruption of pregnancy in mice infected with Toxoplasma gondii during early pregnancy. Infect. immun. 85, https://doi.org/10.1128/ IAl.00257-17 (2017).

14. Andrade, W. A. et al. Combined action of nucleic acid-sensing Toll-like receptors and TLR11/TLR12 heterodimers imparts resistance to Toxoplasma gondii in mice. Cell Host Microbe. 13, 42-53 (2013).

15. Golding, H. et al. Inhibition of HIV-1 infection by a CCR5-binding cyclophilin from Toxoplasma gondii. Blood 102, 3280-3286 (2003).

16. Safronova, A. et al. Alarmin S100A11 initiates a chemokine response to the human pathogen Toxoplasma gondii. Nat. Immunol. 20, 64-72 (2019).

17. Spits, H. et al. Innate lymphoid cells - a proposal for uniform nomenclature. Nat. Rev. Immunol. 13, 145-149 (2013).

18. Spits, H., Bernink, J. H. \& Lanier, L. NK cells and type 1 innate lymphoid cells: partners in host defense. Nat. Immunol. 17, 758-764 (2016).

19. Klose, C. S. \& Artis, D. Innate lymphoid cells as regulators of immunity, inflammation and tissue homeostasis. Nat. Immunol. 17, 765-774 (2016).

20. Klose, C. S. N. et al. Differentiation of type 1 ILCS from a common progenitor to all helper-like innate lymphoid cell lineages. Cell 157, 340-356 (2014).

21. Wagage, S. et al. The Group 3 innate lymphoid cell defect in aryl hydrocarbon receptor deficient mice is associated with $T$ cell hyperactivation during intestinal infection. PLOS ONE 10, e0128335 (2015).

22. Philip, R. \& Epstein, L. B. Tumour necrosis factor as immunomodulator and mediator of monocyte cytotoxicity induced by itself, gamma-interferon and interleukin-1. Nature 323, 86-89 (1986).

23. Deckert-Schluter, M., Albrecht, S., Hof, H., Wiestler, O. D. \& Schluter, D. Dynamics of the intracerebral and splenic cytokine mRNA production in Toxoplasma gondii-resistant and -susceptible congenic strains of mice. Immunology 85, 408-418 (1995).

24. Nagineni, C. N., Pardhasaradhi, K, Martins, M. C., Detrick, B. \& Hooks, J. J. Mechanisms of interferon-induced inhibition of Toxoplasma gondii replication in human retinal pigment epithelial cells. Infect. Immun. 64, 4188-4196 (1996).

25. Chang, H. R., Grau, G. E. \& Pechere, J. C. Role of TNF and IL-1 in infections with Toxoplasma gondii. Immunology 69, 33-37 (1990).

26. Gazzinelli, R. T., Hieny, S., Wynn, T. A., Wolf, S. \& Sher, A. Interleukin 12 is required for the T-lymphocyte-independent induction of interferon gamma by an intracellular parasite and induces resistance in T-cell-deficient hosts. Proc. Natl Acad. Sci. USA 90, 6115-6119 (1993).

27. Hunter, C. A., Subauste, C. S., Van Cleave, V. H. \& Remington, J. S. Production of gamma interferon by natural killer cells from Toxoplasma gondii-infected SCID mice: regulation by interleukin-10, interleukin-12, and tumor necrosis factor alpha. Infect. Immun. 62, 2818-2824 (1994).

28. Askenase, M. H. et al. Bone-marrow-resident NK cells prime monocytes for regulatory function during infection. Immunity 42, 1130-1142 (2015). 
29. Scharton-Kersten, T., Nakajima, H., Yap, G., Sher, A. \& Leonard, W. J. Infection of mice lacking the common cytokine receptor gamma-chain (gamma(c)) reveals an unexpected role for CD4+ T lymphocytes in early IFN-gammadependent resistance to Toxoplasma gondii. J. Immunol. (Baltimore, MD: 1950) 160, 2565-2569 (1998).

30. Sturge, C. R. et al. TLR-independent neutrophil-derived IFN-gamma is important for host resistance to intracellular pathogens. Proc. Natl Acad. Sci. USA 110, 10711-10716 (2013).

31. Kawabe, T. et al. Memory-phenotype CD4(+) T cells spontaneously generated under steady-state conditions exert innate $\mathrm{TH} 1$-like effector function. Sci. Immunol. 2, https://doi.org/10.1126/sciimmunol.aam9304 (2017).

32. Scharton-Kersten, T. M., Yap, G., Magram, J. \& Sher, A. Inducible nitric oxide is essential for host control of persistent but not acute infection with the intracellular pathogen Toxoplasma gondii. J. Exp. Med. 185, 1261-1273 (1997).

33. Khan, I. A., Schwartzman, J. D., Matsuura, T. \& Kasper, L. H. A dichotomous role for nitric oxide during acute Toxoplasma gondii infection in mice. Proc. Natl Acad. Sci. USA 94, 13955-13960 (1997).

34. Schluter, D. et al. Inhibition of inducible nitric oxide synthase exacerbates chronic cerebral toxoplasmosis in Toxoplasma gondii-susceptible C57BL/6 mice but does not reactivate the latent disease in T. gondii-resistant BALB/C mice. J. Immunol. (Baltimore, MD: 1950) 162, 3512-3518 (1999).

35. Murray, H. W. et al. Role of tryptophan degradation in respiratory burstindependent antimicrobial activity of gamma interferon-stimulated human macrophages. Infect. Immun. 57, 845-849 (1989).

36. Daubener, W. et al. Anti-parasitic effector mechanisms in human brain tumor cells: role of interferon-gamma and tumor necrosis factor-alpha. Eur. J. Immunol. 26, 487-492 (1996).

37. Pfefferkorn, E. R. Interferon gamma blocks the growth of Toxoplasma gondii in human fibroblasts by inducing the host cells to degrade tryptophan. Proc. Natl Acad. Sci. USA 81, 908-912 (1984).

38. Gupta, S. L. et al. Antiparasitic and antiproliferative effects of indoleamine 2,3dioxygenase enzyme expression in human fibroblasts. Infect. Immun. 62 2277-2284 (1994).

39. Zlotnik, A. \& Yoshie, O. Chemokines: a new classification system and their role in immunity. Immunity 12, 121-127 (2000).

40. Ma, J. S. et al. Selective and strain-specific NFAT4 activation by the Toxoplasma gondii polymorphic dense granule protein GRA6. J. Exp. Med. 211, 2013-2032 (2014).

41. Getts, D. R. et al. Ly6c+ "inflammatory monocytes" are microglial precursors recruited in a pathogenic manner in West Nile virus encephalitis. J. Exp. Med. 205, 2319-2337 (2008)

42. Sasai, M. et al. Essential role for GABARAP autophagy proteins in interferoninducible GTPase-mediated host defense. Nat. Immunol. 18, 899-910 (2017)

43. Biswas, A. et al. Ly6C(high) monocytes control cerebral toxoplasmosis. J. Immunol. (Baltimore, MD: 1950) 194, 3223-3235 (2015).

44. Oldenhove, G. et al. Decrease of Foxp3+ Treg cell number and acquisition of effector cell phenotype during lethal infection. Immunity 31, 772-786 (2009).

45. Stumhofer, J. S. et al. Interleukin 27 negatively regulates the development of interleukin 17-producing $T$ helper cells during chronic inflammation of the central nervous system. Nat. Immunol. 7, 937-945 (2006).

46. Yoshida, H. \& Hunter, C. A. The immunobiology of interleukin-27. Annu. Rev. Immunol. 33, 417-443 (2015).

47. Sabat, R. IL-10 family of cytokines. Cytokine Growth Factor Rev. 21, 315-324 (2010).

48. Gazzinelli, R. T., Oswald, I. P., James, S. L. \& Sher, A. IL-10 inhibits parasite killing and nitrogen oxide production by IFN-gamma-activated macrophages. J. Immunol. (Baltimore, MD: 1950) 148, 1792-1796 (1992).

49. Burke, J. M., Roberts, C. W., Hunter, C. A., Murray, M. \& Alexander, J. Temporal differences in the expression of mRNA for IL-10 and IFN-gamma in the brains and spleens of C57BL/10 mice infected with Toxoplasma gondii. Parasite Immunol. 16, 305-314 (1994).

50. Swierczynski, B. et al. Inhibitory activity of anti-interleukin-4 and antiinterleukin-10 antibodies on Toxoplasma gondii proliferation in mouse peritoneal macrophages cocultured with splenocytes from infected mice. Parasitol. Res. 86, 151-157 (2000).

51. Gazzinelli, R. T. et al. In the absence of endogenous IL-10, mice acutely infected with Toxoplasma gondii succumb to a lethal immune response dependent on CD4+ T cells and accompanied by overproduction of IL-12, IFN-gamma and TNF-alpha. J. Immunol. (Baltimore, MD: 1950) 157, 798-805 (1996).
52. Neyer, L. E. et al. Role of interleukin-10 in regulation of T-cell-dependent and T-cell-independent mechanisms of resistance to Toxoplasma gondii. Infect. Immun. 65, 1675-1682 (1997)

53. Jeong, Y. I., Hong, S. H., Cho, S. H., Park, M. Y. \& Lee, S. E. Induction of IL-10producing regulatory $\mathrm{B}$ cells following Toxoplasma gondii infection is important to the cyst formation. Biochem. Biophys. Rep. 7, 91-97 (2016).

54. Jankovic, D. et al. Conventional T-bet(+)Foxp3(-) Th1 cells are the major source of host-protective regulatory $\mathrm{IL}-10$ during intracellular protozoan infection. J. Exp. Med. 204, 273-283 (2007).

55. Koch, M. A. et al. The transcription factor T-bet controls regulatory $T$ cell homeostasis and function during type 1 inflammation. Nat. Immunol. 10 595-602 (2009).

56. Neumann, C. et al. Role of Blimp-1 in programing Th effector cells into IL-10 producers. J. Exp. Med. 211, 1807-1819 (2014).

57. Nascimento, D. C. et al. IL-33 contributes to sepsis-induced long-term immunosuppression by expanding the regulatory $\mathrm{T}$ cell population. Nat. Commun. 8, 14919 (2017).

58. Sher, A., Denkers, E. Y. \& Gazzinelli, R. T. Induction and regulation of host cellmediated immunity by Toxoplasma gondii. Ciba Found. Symp. 195, 95-104 (1995). discussion 104-109.

59. Taylor, G. A., Feng, C. G. \& Sher, A. p47 GTPases: regulators of immunity to intracellular pathogens. Nat. Rev. Immunol. 4, 100-109 (2004).

60. Boehm, U. et al. Two families of GTPases dominate the complex cellular response to IFN-gamma. J. Immunol. (Baltimore, MD: 1950) 161, 6715-6723 (1998).

61. Bekpen, C. et al. The interferon-inducible p47 (IRG) GTPases in vertebrates: loss of the cell autonomous resistance mechanism in the human lineage. Genome Biol. 6, R92 (2005).

62. Sorace, J. M., Johnson, R. J., Howard, D. L. \& Drysdale, B. E. Identification of an endotoxin and IFN-inducible CDNA: possible identification of a novel protein family. J. Leukoc. Biol. 58, 477-484 (1995).

63. Taylor, G. A. et al. Identification of a novel GTPase, the inducibly expressed GTPase, that accumulates in response to interferon gamma. J. Biol. Chem 271, 20399-20405 (1996)

64. Taylor, G. A. et al. The inducibly expressed GTPase localizes to the endoplasmic reticulum, independently of GTP binding. J. Biol. Chem. 272, 10639-10645 (1997).

65. Zerrahn, J., Schaible, U. E., Brinkmann, V., Guhlich, U. \& Kaufmann, S. H. The IFN-inducible Golgi- and endoplasmic reticulum-associated 47-kDa GTPase $\| G P$ is transiently expressed during listeriosis. J. Immunol. (Baltimore, MD: 1950) 168, 3428-3436 (2002)

66. Zhao, Y. O., Konen-Waisman, S., Taylor, G. A., Martens, S. \& Howard, J. C. Localisation and mislocalisation of the interferon-inducible immunity-related GTPase, Irgm1 (LRG-47) in mouse cells. PLOS ONE 5, e8648 (2010).

67. Carlow, D. A., Marth, J., Clark-Lewis, I. \& Teh, H. S. Isolation of a gene encoding a developmentally regulated $T$ cell-specific protein with a guanine nucleotide triphosphate-binding motif. J. Immunol. (Baltimore, MD: 1950) 154, 1724-1734 (1995).

68. Lafuse, W. P., Brown, D., Castle, L. \& Zwilling, B. S. IFN-gamma increases cathepsin $\mathrm{H}$ mRNA levels in mouse macrophages. J. Leukoc. Biol. 57, 663-669 (1995).

69. Hunn, J. P. et al. Regulatory interactions between IRG resistance GTPases in the cellular response to Toxoplasma gondii. EMBO J. 27, 2495-2509 (2008).

70. Khaminets, A. et al. Coordinated loading of IRG resistance GTPases on to the Toxoplasma gondii parasitophorous vacuole. Cell. Microbiol. 12, 939-961 (2010).

71. Henry, S. C. et al. Balance of Irgm protein activities determines IFN-gammainduced host defense. J. Leukoc. Biol. 85, 877-885 (2009).

72. Haldar, A. K. et al. IRG and GBP host resistance factors target aberrant, "nonself" vacuoles characterized by the missing of "self" IRGM proteins. PLoS Pathog. 9, e1003414 (2013)

73. Collazo, C. M. et al. Inactivation of LRG-47 and IRG-47 reveals a family of interferon gamma-inducible genes with essential, pathogen-specific roles in resistance to infection. J. Exp. Med. 194, 181-188 (2001)

74. Martens, S. et al. Disruption of Toxoplasma gondii parasitophorous vacuoles by the mouse p47-resistance GTPases. PLoS Pathog. 1, e24 (2005).

75. MacMicking, J. D. IFN-inducible GTPases and immunity to intracellular pathogens. Trends Immunol. 25, 601-609 (2004).

76. Kim, B. H. et al. A family of IFN-gamma-inducible 65-kD GTPases protects against bacterial infection. Science (New York, NY) 332, 717-721 (2011). 
77. Selleck, E. M. et al. Guanylate-binding protein 1 (Gbp1) contributes to cellautonomous immunity against Toxoplasma gondii. PLoS Pathog. 9, e1003320 (2013)

78. Yamamoto, M. et al. A cluster of interferon- $\gamma$-inducible p65 GTPases plays a critical role in host defense against Toxoplasma gondii. Immunity 37, 302-313 (2012).

79. Degrandi, D. et al. Murine Guanylate Binding Protein 2 (mGBP2) controls Toxoplasma gondii replication. Proc. Natl Acad. Sci. USA 110, 294-299 (2013).

80. Kravets, E. et al. The GTPase activity of murine guanylate-binding protein 2 (mGBP2) controls the intracellular localization and recruitment to the parasitophorous vacuole of Toxoplasma gondii. J. Biol. Chem. 287, 27452-27466 (2012)

81. Perrin, A. J., Jiang, X., Birmingham, C. L., So, N. S. \& Brumell, J. H. Recognition of bacteria in the cytosol of mammalian cells by the ubiquitin system. Curr. Biol. 14, 806-811 (2004)

82. Selleck, E. M. et al. A noncanonical autophagy pathway restricts Toxoplasma gondii growth in a strain-specific manner in IFN-gamma-activated human cells. mBio 6, e01157-01115 (2015).

83. Lee, $Y$. et al. p62 plays a specific role in interferon-gamma-induced presentation of a toxoplasma vacuolar antigen. Cell Rep. 13, 223-233 (2015).

84. Clough, B. et al. K63-linked ubiquitination targets Toxoplasma gondii for endo-lysosomal destruction in IFNgamma-stimulated human cells. PLoS Pathog. 12, e1006027 (2016).

85. Foltz, C. et al. TRIM21 is critical for survival of Toxoplasma gondii infection and localises to GBP-positive parasite vacuoles. Sci. Rep. 7, 5209 (2017).

86. Encheva, V., Foltz, C., Snijders, A. P. \& Frickel, E. M. Murine Gbp1 and Gbp2 are ubiquitinated independent of Toxoplasma gondii infection. BMC Res. Notes 11, 166 (2018).

87. Haldar, A. K. et al. Ubiquitin systems mark pathogen-containing vacuoles as targets for host defense by guanylate binding proteins. Proc. Natl Acad. Sci. USA 112, E5628-E5637 (2015).

88. Mizushima, N., Yoshimori, T. \& Ohsumi, Y. The role of Atg proteins in autophagosome formation. Annu. Rev. Cell Dev. Biol. 27, 107-132 (2011).

89. Slobodkin, M. R. \& Elazar, Z. The Atg8 family: multifunctional ubiquitin-like key regulators of autophagy. Essays Biochem. 55, 51-64 (2013).

90. Sumpter, R. Jr. \& Levine, B. Autophagy and innate immunity: triggering, targeting and tuning. Semin. Cell Dev. Biol. 21, 699-711 (2010).

91. Kudchodkar, S. B. \& Levine, B. Viruses and autophagy. Rev. Med. Virol. 19, 359-378 (2009).

92. Andrade, R. M., Wessendarp, M., Gubbels, M. J., Striepen, B. \& Subauste, C. S. CD40 induces macrophage anti-Toxoplasma gondii activity by triggering autophagy-dependent fusion of pathogen-containing vacuoles and lysosomes. J. Clin. Investig. 116, 2366-2377 (2006).

93. Park, S. et al. Targeting by AutophaGy proteins (TAG): targeting of IFNGinducible GTPases to membranes by the LC3 conjugation system of autophagy. Autophagy 12, 1153-1167 (2016).

94. Hanada, T. et al. The Atg12-Atg5 conjugate has a novel E3-like activity for protein lipidation in autophagy. J. Biol. Chem. 282, 37298-37302 (2007).

95. Choi, J. et al. The parasitophorous vacuole membrane of Toxoplasma gondii is targeted for disruption by ubiquitin-like conjugation systems of autophagy. Immunity 40, 924-935 (2014).

96. Zhao, Z. et al. Autophagosome-independent essential function for the autophagy protein Atg5 in cellular immunity to intracellular pathogens. Cell Host Microbe. 4, 458-469 (2008).

97. Haldar, A. K., Piro, A. S., Pilla, D. M., Yamamoto, M. \& Coers, J. The E2-like conjugation enzyme Atg3 promotes binding of IRG and Gbp proteins to Chlamydia- and Toxoplasma-containing vacuoles and host resistance. PLoS ONE 9, e86684 (2014).

98. Pilla, D. M. et al. Guanylate binding proteins promote caspase-11-dependent pyroptosis in response to cytoplasmic LPS. Proc. Natl Acad. Sci. USA 111, 6046-6051 (2014).

99. Finethy, R. et al. Guanylate binding proteins enable rapid activation of canonical and noncanonical inflammasomes in Chlamydia-infected macrophages. Infect. Immun. 83, 4740-4749 (2015).

100. Man, S. M. et al. The transcription factor IRF1 and guanylate-binding proteins target activation of the AIM2 inflammasome by Francisella infection. Nat. Immunol. 16, 467-475 (2015).

101. Meunier, E. et al. Guanylate-binding proteins promote activation of the AlM2 inflammasome during infection with Francisella novicida. Nat. Immunol. 16 476-484 (2015).

102. Man, S. M. et al. IRGB10 liberates bacterial ligands for sensing by the AIM2 and Caspase-11-NLRP3 inflammasomes. Cell 167, 382-396 (2016). e317.

103. Santos, J. C. et al. LPS targets host guanylate-binding proteins to the bacteria outer membrane for non-canonical inflammasome activation. EMBO J. 37 https://doi.org/10.15252/embj.201798089 (2018).

104. Li, P. et al. Ubiquitination and degradation of GBPs by a Shigella effector to suppress host defence. Nature 551, 378-383 (2017).

105. Wandel, M. P. et al. GBPs inhibit motility of Shigella flexneri but are targeted for degradation by the bacterial ubiquitin ligase IpaH9.8. Cell Host Microbe. 22, 507-518, e505 (2017) 